\title{
Uterine Ligament Adenocarcinoma
}

National Cancer Institute

\section{Source}

National Cancer Institute. Uterine Ligament Adenocarcinoma. NCI Thesaurus. Code C40135.

A rare adenocarcinoma that arises from the uterine ligament. 